\title{
Coronal Mass Ejections and Angular Momentum Loss in Young Stars
}

\author{
Alicia N. Aarnio ${ }^{1}$, Keivan G. Stassun ${ }^{2,3}$ and Sean P. Matt ${ }^{4}$ \\ ${ }^{1}$ Dept. of Astronomy, University of Michigan, Ann Arbor, MI, 48109, USA \\ email: aarnio@umich.edu \\ ${ }^{2}$ Dept. of Physics \& Astronomy, Vanderbilt University, Nashville, TN, 37235, USA \\ ${ }^{3}$ Dept. of Physics, Fisk University, Nashville, TN, 37208 USA \\ ${ }^{4}$ School of Physics, University of Exeter, \\ Stocker Road, Exeter, EX4 4QL, UK
}

\begin{abstract}
In our own solar system, the necessity of understanding space weather is readily evident. Fortunately for Earth, our nearest stellar neighbor is relatively quiet, exhibiting activity levels several orders of magnitude lower than young, solar-type stars. In protoplanetary systems, stellar magnetic phenomena observed are analogous to the solar case, but dramatically enhanced on all physical scales: bigger, more energetic, more frequent. While coronal mass ejections (CMEs) could play a significant role in the evolution of protoplanets, they could also affect the evolution of the central star itself. To assess the consequences of prominence eruption/CMEs, we have invoked the solar-stellar connection to estimate, for young, solar-type stars, how frequently stellar CMEs may occur and their attendant mass and angular momentum loss rates. We will demonstrate the necessary conditions under which CMEs could slow stellar rotation.
\end{abstract}

Keywords. Sun: flares, Sun: coronal mass ejections, stars: activity, stars: flare, stars: evolution, stars: rotation, stars: planetary systems: protoplanetary disks

\section{Introduction}

On young stars, we observe flares hundreds to ten thousand times more energetic and frequent than solar flares. Along with energy scales greater by orders of magnitude, we also observe physical scales far greater than in the solar case: while solar prominences soar around $1 \mathrm{R}_{\odot}$ above the solar surface and CMEs launch from similar radii in the Sun's atmosphere, magnetic structures on T Tauri Stars (TTS, young solar analogs)post-flare loops and prominences-can extend tens of stellar radii from the star's surface. The discovery of such large magnetic structures arose from solar-stellar analogy, applying solar flare models to the X-ray light curve data from young stars (e.g., Reale et al. 1998).

In characterizing the solar-stellar connection, overwhelming evidence has been found in support of the idea that the fundamental physics of magnetic reconnection is the same, despite differences in stellar parameters (e.g., mass, radius, $B$, age). As such, we approach analysis of young stars' flares and CMEs under this supposition, and aim to assess how the physical properties of these events-and their frequency-may scale accordingly with stellar parameters. Ultimately, we seek to understand the consequences of exoplanetary space weather on protostellar systems and their forming planets.

\section{Estimating Angular Momentum Loss via Stellar CMEs}

The inference of magnetic loops many stellar radii in extent (Favata et al. 2005; hereafter F05) inspired three questions: one, are these loops interacting with circumstellar disks? In Aarnio et al. (2010), we did not find evidence for this. Second, if there is not 


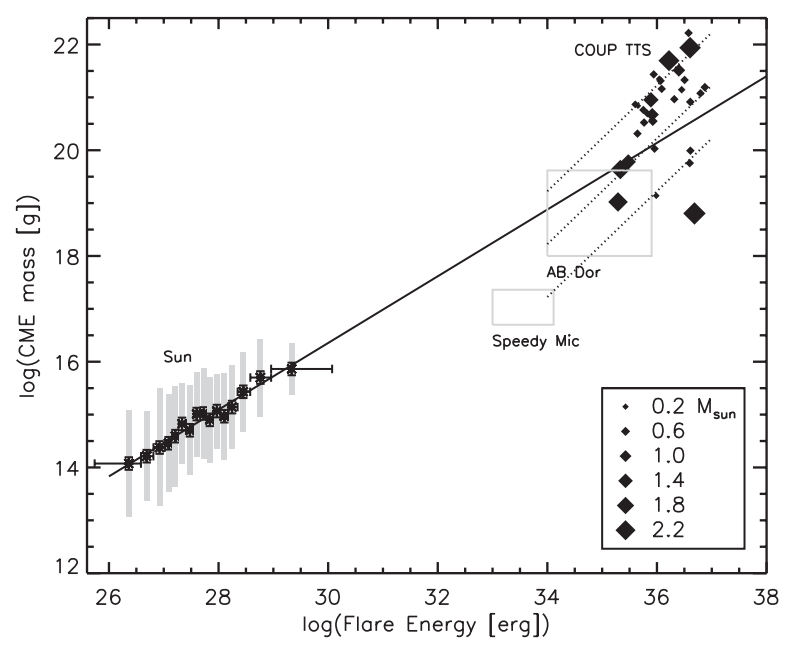

Figure 1. CME mass/flare energy relationship of AA11 shown with TTS post-flare loop masses (black diamonds) derived in AA12 plotted as a function of the flare's energy. Point size denotes the mass of the star on which the flare was observed. Black dotted lines show predicted post-flare stellar loop masses (Eqn. 2.1 and discussion in text) for a range of observed densities from $10^{10}-10^{12}$ $\mathrm{cm}^{-3}$ and assuming a confining field strength of 50G. Gray, solid-lined boxes denote the range of observed $\mathrm{X}$-ray flare energies (Maggio et al. 2000, Collier Cameron et al. 1988) and cool $\mathrm{H} \alpha$ prominence mass estimates (Collier Cameron \& Robinson, 1989a,b) for AB Dor and Speedy Mic, 20-50Myr K dwarfs.

a star-disk link, how do the loops remain stable for the multiple rotation periods over which the X-ray flares are observed to decay? We showed in Aarnio et al. (2012) that when modeled as hot prominences, the addition of a scaled-up wind consistent with TTS observations provided sufficient support for the loops to be stable. Finally, here (and in Aarnio, Matt, \& Stassun, 2012; hereafter AA12) we address the third question of what happens when stability is lost: at many stellar radii, is the specific angular momentum shed significant enough to slow stellar rotation?

In order to estimate the effects of eruptive prominences and stellar CMEs on the rotation of young stars, we must procure two ingredients: the mass lost via these events, and their frequency of occurrence. Despite ongoing and historical efforts to observe stellar CMEs, we lack definitive detections and thus frequency distributions. It is known that at times, magnetic reconnection on the Sun will produce both a flare and an associated CME; for stars, the flare is the observable quantity, and so we characterize stellar CME frequency by using stellar flare frequency as a proxy.

In Fig. 1, we show our solar flare energy/CME mass relationship (Aarnio et al. 2011, hereafer AA11) extrapolated up to the energies of young stellar flares. We calculate loop masses for the 32 "superflaring" stars from the Chandra Orion Ultradeep Project (Getman et al. 2005) from the parameters reported by F05. Interestingly, these loop masses are close in parameter space to the extrapolated solar relationship. This is perhaps unsurprising, as the plasma confined in a post-flare loop has properties which relate to the energy of the flare. In AA11, we found that for associated flares and CMEs, that is to say, flares and CMEs which likely originated from a shared magnetic reconnection event, the CME mass and flare energy were related. As such, the post-flare loop mass and mass of an associated CME should then also be related.

From Shibata \& Yokoyama (2002), we can estimate the total energy released by a flare is related to the total magnetic energy in a flare loop:

$$
E_{\text {mag }}=\frac{B^{2} L^{3}}{8 \pi} .
$$

If we substitute the loop volume expressed in terms of mass (i.e., $L^{3} \sim V=m_{\text {loop }} / \rho$ ), we find a relationship between the total flare energy and the mass confined in the magnetic loop (dashed, parallel lines in Fig. 1). 


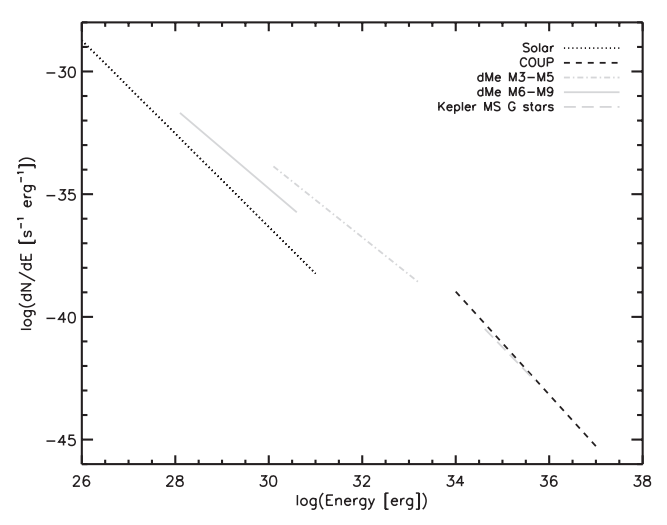

Figure 2. Flare frequencies for the Sun, M dwarfs (Hilton et al. 2011), TTS in the ONC (Albacete Colombo et al. 2007), and active, main sequence G stars from Kepler (Maehara et al. 2012). Interestingly, the active $\mathrm{G}$ stars are almost indistinguishable from the TTS. Note the solar and TTS frequencies are derived from $\mathrm{X}$-ray flare data, while the $\mathrm{M}$ dwarf and G stars are optical flare frequencies.

Here, to be consistent with the analysis of F05, we assume an Euclidian loop filling but do note that recent solar X-ray flare imaging has indicated that a fractal scaling of $\mathrm{V}(\mathrm{L}) \propto \mathrm{L}^{2.4}$ is likely a more accurate characterization (e.g., Aschwanden, Stern, \& Güdel, 2008).

It is remarkable that the post-flare loop masses even lie near the extrapolated solar CME mass/flare energy relationship, several orders of magnitude away in parameter space. In Fig. 1, we have also shown representative ranges of X-ray flare energy and prominence mass for two $\mathrm{K}$ dwarfs intermediate in age to the TTS sample and the Sun; with an eruptive prominence thought to be the core of a CME, these mass ranges likely represent lower limits on the range of CME masses on these stars. In the following calculations, to represent a fiducial TTS case, we will simply extrapolate the solar relationship to generate a stellar CME mass distribution.

In Fig. 2, we show the frequency distributions for flares observed on the Sun, TTS, $\mathrm{M}$ dwarfs, and active, main sequence G stars. For this work, we use the TTS frequency distribution. Clearly, not all CMEs are flare-associated, nor are all flares CME-associated; AA11 found, however, that the association fraction increases with increasing flare energy, so for young stars for which we observe flares several orders of magnitude more energetic than in the solar case, we simply assume this association fraction to be of order unity.

\subsection{Angular momentum loss}

In AA12, we extrapolate the solar CME mass/flare energy relationship (Fig. 1) to TTS flare energies and frequencies (Fig. 2) and construct a CME frequency distribution as a function of CME mass. Given the observational completeness limits on the flare distributions that went into the CME distribution, we derive lower and upper limits on the mass loss rate by empirical (integrating the distribution) and analytical (integrating a fit to the distribution) means. The range of mass loss rates we estimate for the TTS case is $10^{-12}-10^{-9} \mathrm{M}_{\odot} \mathrm{yr}^{-1}$.

To assess the torque applied against stellar rotation by these CMEs, we apply stellar wind models with mass loss rates set as determined above. Given the episodic nature of CMEs, we included an efficiency parameter to account for the fact that steady-state winds are more efficient at removing angular momentum than "clumpy" winds (cf. AA12 and references therein). We adopt a dipolar field with strength $600 \mathrm{G}$, consistent with observations of TTS fields, and allow the stellar radius to contract as stellar evolution models predict.

In a protostellar system, multiple torques act simultaneously to spin up and spin down the star. In this analysis, we compared spin up due to contraction and spin down due to mass loss from stellar CMEs to see if, at any point in the pre-main sequence our fiducial 
TTS could have its rotation slowed due to CMEs. Comparing parameters of efficiency and the range of mass loss rates we calculated, it became clear that only towards the end of the pre-main sequence phase (ages $\gtrsim 6$ Myr) could a very efficient, high CME mass loss rate begin to counteract spin up from contraction. We have left out factors such as spin up from accretion and mass loss via stellar wind; Matt \& Pudritz (2005) explore these two torques in depth and the necessary conditions for an accretion powered stellar wind to slow stellar rotation.

\section{Discussion}

We have shown that for young, solar-type stars, spin down due to CMEs might play a significant role in stellar rotation evolution after the star has ceased accreting. Our Figs. 1 and 2 illustrate a critical selection effect in performing this kind of calculation: we only have data for the most active young stars, or the star conveniently located at 1 AU. There is a dearth of data for older, less active stars, and we suggest that filling in the gaps in flare X-ray energy could trace age evolution in these parameter spaces. The addition of data from the $\sim 20-50$ Myr old K dwarfs in Fig. 1 hints at this, but more data are needed to conclusively show age dependence. In both figures, we have taken care to specify the masses of the stars involved: how would evolution with stellar age look in these parameter spaces as a function of stellar mass? While the fundamental physics are the same, the scaling could change, and the ramifications certainly would. For low-mass stars in particular, high activity levels are observed for longer fractions of the stars' lives; this could have grave implications for exoplanets as these stars' habitable zones could be within range of extreme exo-weather.

\section{Acknowledgements}

A. N. A. thanks K. Shibata for helpful discussion regarding stellar post-flare loops.

\section{References}

Aarnio, A. N., Matt, S. P., \& Stassun, K. G. 2012, ApJ, 760, 9

Aarnio, A. N., Llama, J., Jardine, M., \& Gregory, S. G. 2012, MNRAS, 421, 1797

Aarnio, A. N., Stassun, K. G., Hughes, W. J., \& McGregor, S. L. 2011, Solar Phys., 268, 195

Aarnio, A. N., Stassun, K. G., \& Matt, S. P. 2010, ApJ, 717, 93

Albacete Colombo, J. F., Flaccomio, E., Micela, G., Sciortino, S., \& Damiani, F. 2007, A\&̊A, 464,211

Aschwanden, M. J., Stern, R. A., \& Güdel, M. 2008, ApJ 672, 659

Collier Cameron, A. \& Robinson, R. D. 1989, MNRAS, 238, 657

Collier Cameron, A. \& Robinson, R. D. 1989, MNRAS, 236, 57

Collier Cameron, A., Bedford, D. K., Rucinski, S. M., Vilhu, O., \& White, N. E. 1988, MNRAS, 231,131

Dunstone, N. J., Collier Cameron, A., Barnes, J. R., \& Jardine, M. 2006, MNRAS, 373, 1308

Favata, F., Flaccomio, E., Reale, F., Micela, G., Sciortino, S., Shang, H., Stassun, K. G., \& Feigelson, E. D. 2005, ApJS, 160, 469

Getman, K. V. et al. 2005, ApJS, 160, 319

Hilton, E. J., Hawley, S. L., Kowalski, A. F., \& Holtzman, J. 2011, ASPC 16th Cambridge Workshop on Cool Stars, Stellar Systems, and the Sun, 448, 197

Maehara, H., Shibayama, T., Notsu, S., Notsu, Y., Nagao, T., Kusaba, S., Honda, S., Nogami, D., \& Shibata, K. 2012, Nature, 485, 478

Maggio, A., Pallavicini, R., Reale, F., \& Tagliaferri, G. 2000, A\&A, 356, 627

Matt, S. P. \& Pudritz, R. E. 2005, ApJL, 632, L135

Reale, F. \& Micela, G. 1998, $A \& A$, 334, 1028

Shibata, K. \& Yokoyama, T. 2002, ApJ, 577, 422 